\title{
Athlete Robot with Applied Human Muscle Activation Patterns for Bipedal Running
}

\author{
Ryuma Niiyama \\ Department of Mechano-Informatics \\ University of Tokyo \\ Tokyo, Japan \\ Email: niiyama@isi.imi.i.u-tokyo.ac.jp
}

\author{
Satoshi Nishikawa \\ Graduate School of \\ Interdisciplinary Information Studies \\ University of Tokyo \\ Tokyo, Japan
}

\author{
Yasuo Kuniyoshi \\ Department of Mechano-Informatics \\ University of Tokyo \\ Tokyo, Japan \\ Email: kuniyosh@isi.imi.i.u-tokyo.ac.jp
}

Email: nisikawa@isi.imi.i.u-tokyo.ac.jp

\begin{abstract}
The essential component of legged locomotion is control of the ground reaction force. To understand the role of the musculoskeletal body in dynamic locomotion, we investigate bipedal running using a musculoskeletal "Athlete Robot". The configuration of the muscles in the robot is compatible with the human. The spring-like property of the human lower leg during running is modeled as an elastic blade foot based on findings from biomechanics. The motor command of the robot is represented by time series data of muscle activation. The muscle activation patterns are determined from numerical calculation using a model of the musculoskeletal leg based on the measurement of muscle activity and kinetic data of the human movements. In the simulation results, the robot runs 8 steps with a speed of $3 \mathrm{~m} / \mathrm{s}$. We also demonstrate that the real bipedal robot is able to run for several steps.
\end{abstract}

\section{INTRODUCTION}

In legged locomotion, the ground reaction force provides thrust force to move forward and moment to maintain the posture. The Raibert's hopping robot shows essentials in legged locomotion using a simple mechanism of telescopic leg [1]. However, the relationship between the method to control simplified model and the motor command used in musculoskeletal human body (Fig.1) is still not clear.

There are several humanoid robots capable of generating dynamic bipedal running [2], [3]. The standard humanoid robot uses servo controller to follow the desired joint angles generated from a planning of the footprint and ZMP position. The method can only control ground reaction force indirectly through the geometric condition of the robot. In addition, tendon-like elastic element is incompatible with precise angle control.

In contrast, animals perform dynamic legged locomotion with their musculoskeletal system, which has no sensor for absolute joint angles, and has viscoelasticity in muscle-tendon tissues [4]. To investigate the role of musculoskeletal force control in legged locomotion, we focused on bipedal running with musculoskeletal robot driven by pneumatic artificial muscles. A force control plays an important part in support phase of bipedal running. We focused on the musculoskeletal force control behind the measured ground reaction force. We suppose that the observed complex time series data of joint angles in running motion originate from relatively simple muscle

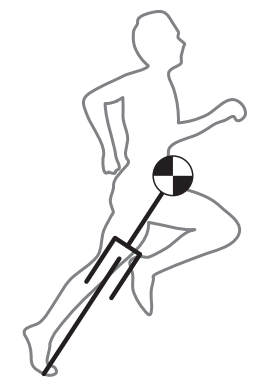

(a) inverted pendulum

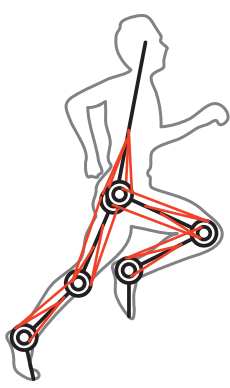

(b) musculoskeletal

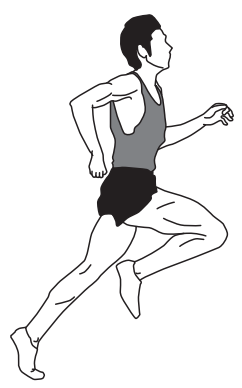

(c) human body
Fig. 1. Various models for human running.

activation patterns. Our hypothesis is that the musculoskeletal force control eases the motion generation in adaptive running motion, and tolerates elasticity of the mechanism.

A few challenges in force control of legged locomotion with humanoid robot have been conducted. A passive balancing on the uneven ground with torque control is studied using SARCOS full-body humanoid robot driven by hydraulic actuators [5]. A hydraulic-powered biped PETMAN robot is also expected to control the body posture through force control method also used for quadruped BigDog robot [6].

\section{Artificial Musculoskeletal System}

\section{A. Musculoskeletal Robot}

The musculoskeletal system has unique mechanisms such as viscoelasticity in muscle-tendon complex, mono- and biarticular muscles and tendon-pulley system to perform agile and dynamic movements. The mechanical properties of the muscle-tendon tissue and its function in energy regeneration are much debated issue in biomechanics research. The muscletendon unit is kind of direct drive mechanism which generate joint torque without any reductions.

On the other hand, there are a few application of musculoskeletal system to robot in the previous study. The Kotaro and Kojiro robot is the full-scale humanoid robot driven by the number of wire tension actuator [7]. The robot shows the advantages of flexibility derived from redundant layout of actuators and flexible spine structure. The Lucy is also a bipedal 


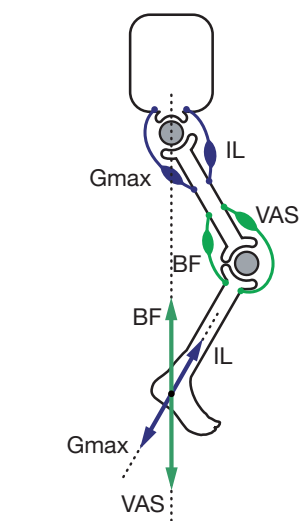

(a) independently driven joints with mono-articular muscles

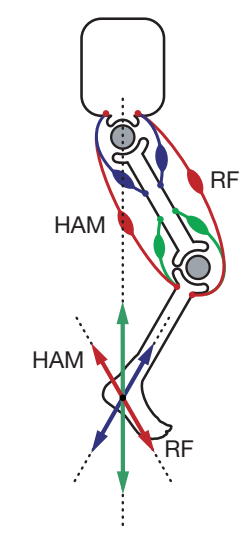

(b) combination of mono-articular muscles and bi-articular muscles
Fig. 2. Bi-articular function in direction of force output.

musculoskeletal robot driven by the antagonistic mechanism with mono-articular pneumatic muscles [8]. They use ZMP based motion planning for walking with joint angle control. The anthropomorphic musculoskeletal robot is proposed [9], which perform the quasi-passive walking, hopping and a few step dash, controlled with just only on/off function of solenoid valves.

\section{B. Muscle Configuration and Force Output}

The bi-articular muscles are those that cross two joints and supply torques at both joints simultaneously. The functions of bi-articular muscles are not known exactly. The previous study shows that the bi-articular muscles can play a role of the force control in the translation of the body center of gravity [10], [11].

The relationships between muscle configuration and force output of the musculoskeletal system are described and studied using robot arm [12], [13].

The statics equation of the musculoskeletal leg shows that the bi-articular muscles provide force output unavailable in independently-driven joint with mono-articular muscle or electric motor (Fig.2).

A musculoskeletal robot with force-controlled mono- and bi-articular muscles are required.

\section{Athlete Robot}

\section{A. Overview}

An "Athlete Robot" is bipedal robot with the artificial musculoskeletal system to perform sprint running (Fig.3). The pneumatic artificial muscles are used for the system. The pneumatic muscle allow dynamic and agile movements for the robot with its property of light-weight and large amount of energy converted in short period of motion. In use of pneumatic actuator driven by pressured air is not suitable for the precise position/angle control application. In contrast, we can control force/torque with relatively ease by the control of the air pressure. We employ custom-made proportional pressure control valve to control the inner pressure of the

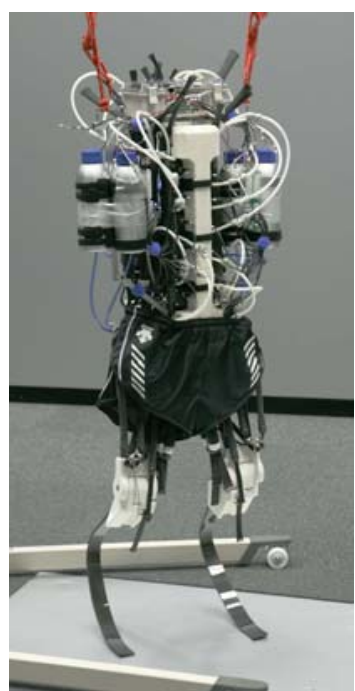

Fig. 3. Musculoskeletal Athlete Robot.

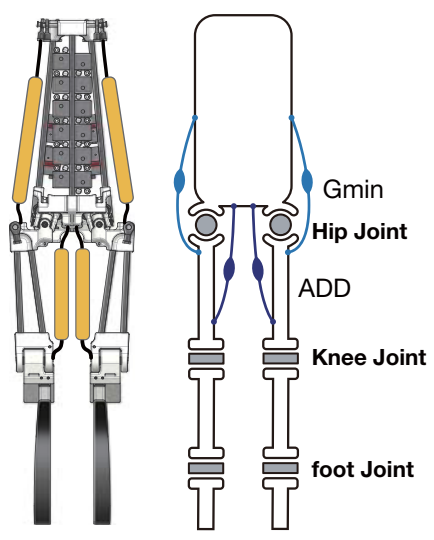

(a) front view

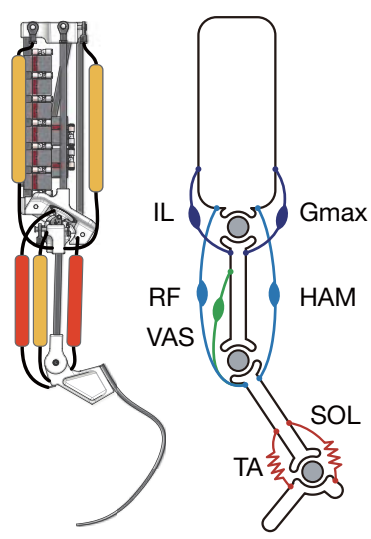

(b) side view
Fig. 4. Layout of the muscles. The symbols are, Gmin: gluteus minimus muscle, ADD: adductor muscles, Gmax: gluteus maximus muscle, IL: iliopsoas muscle, HAM: hamstrings, RF: rectus femoris muscle, VAS: vastus muscles, SOL: soleus muscle, TA: tibialis anterior muscle, respectively.

muscles. The robot with musculoskeletal mechanisms and the controller of the muscle tension force is an appropriate platform for bipedal running with the force control.

\section{B. Muscular System}

The artificial musculoskeletal system of the robot is based on the anatomical structure of the human (Fig.4). We carefully chose the parameters of mono-articular and bi-articular muscles, such as diameter, length, and moment arm of the tendon-pulley unit for each muscles (Fig.5).

The parameters of muscle configuration are decided by consideration of both mathematically obtained parameters using model of musculoskeletal leg and anatomical data of the human. The physiological cross-sectional area: PCSA and mass of the human muscles are reported in the literature in biomechanics [14], [15]. 


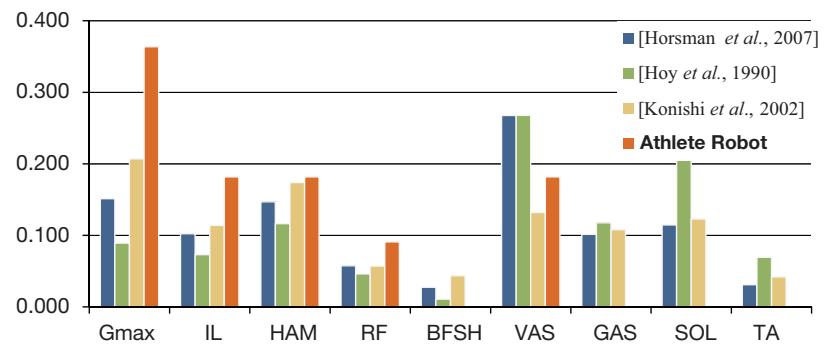

Fig. 5. Proportion of the size of muscles.

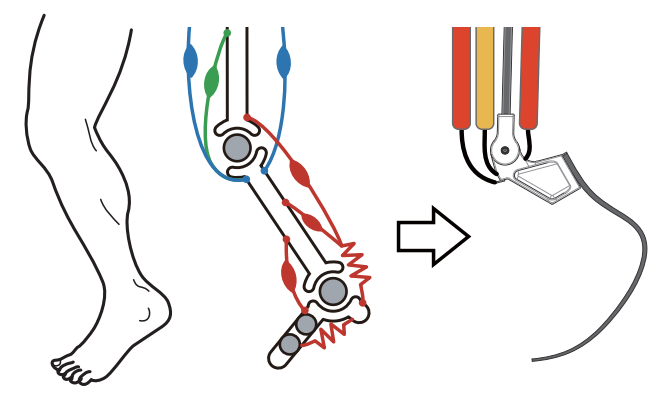

Fig. 6. Musculoskeletal system of the human lower leg and simplified foot as blade spring.

\section{Blade Foot}

The lower leg of the human behaves like a non-linear spring during dynamic locomotion [16], [17]. The muscles in lower leg suppose to be used for fixing a tendon. In other proof, the human athlete wearing spring foot, called "blade runner", could perform sprint running similar to normal people. From these insights, we modeled the human lower leg in sprint running as blade spring (Fig.6).

The physically inserted elastic element has advantages in response to uncertainty of rough terrain and high frequency impact force compared to the software feedback [18]. Though, the elastic component negatively affect to the performance of PID position control, the force control would work successfully.

\section{Musculoskeletal Motor Control}

\section{A. Overview}

The method of musculoskeletal motor control proposed here is consists of measurement of human running and computation of the muscle activation pattern by numerical optimization.

We use SCA (Sparse Coding of Activation) method to represent a motor command [19]. It is an abstract representation of the movements with the simplified muscle activation patterns as opposed to a high-dimensional state of the robot. The patterns are generated by the combination of the appropriate basis functions under the constraint of sparseness. Here, we use a simple step function as a basis function. The activation pattern is divided to $n$ phases with duration time $T_{n}$ (Fig.7).

From the collected kinematic and kinetic data during human running, we divide motor command to four phases in one

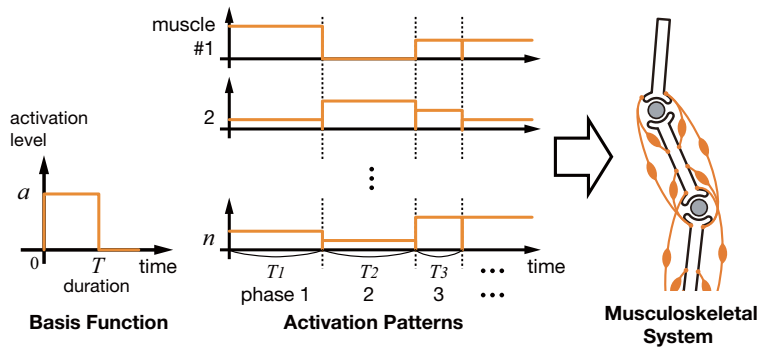

Fig. 7. The SCA method to represent the time series data of the muscle activation.
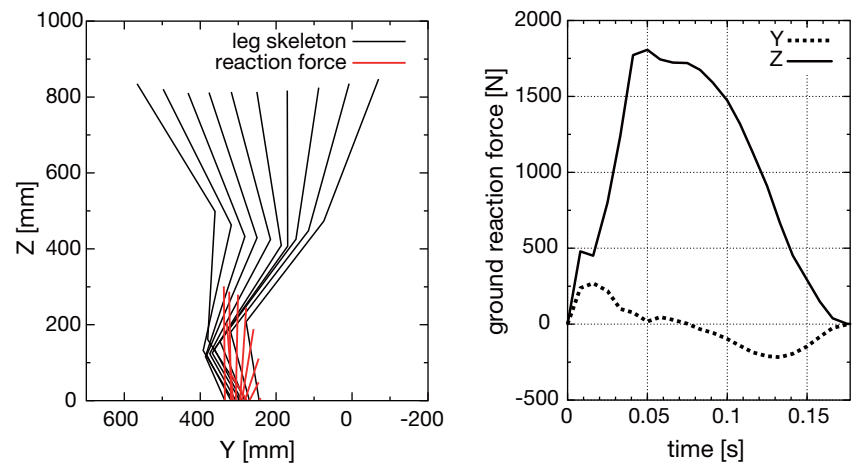

Fig. 8. The direction and magnitude of ground reaction force in human running.

period of running [20]. The phases are phase of support, recovery swing I, recovery swing II and descent foot.

\section{B. Muscle Activation in the Support Phase}

In the support phase, the robot is required to generate a contact force in the dynamically constrained motion. The control of the ground reaction force in the support phase is the inverse problem to compute required muscle tension forces from desired ground reaction force. We propose a method for determining the muscle activation patterns from a measurement of ground reaction force of human running (Fig.8).

Let $\boldsymbol{Q}$ be the generalize force consist of force $\boldsymbol{F}$ and moment $\boldsymbol{M}$ at the contact point between leg and environment (eq.(1)).

$$
Q=\left[\begin{array}{c}
F \\
M
\end{array}\right]
$$

The relationships between force output of the leg $\boldsymbol{Q}$ and tension force $\boldsymbol{f}$ generated by the muscles are described using well-known equation of the duality between differential kinematics and statics as:

$$
\boldsymbol{G}^{T}(\boldsymbol{\theta}) \boldsymbol{f}-\boldsymbol{J}^{T}(\boldsymbol{\theta}) \boldsymbol{Q}=\boldsymbol{O}
$$

Here, a matrix $\boldsymbol{J}(\boldsymbol{\theta})$ is the Jacobian matrix represents the differential relationship between the joint motion and the 


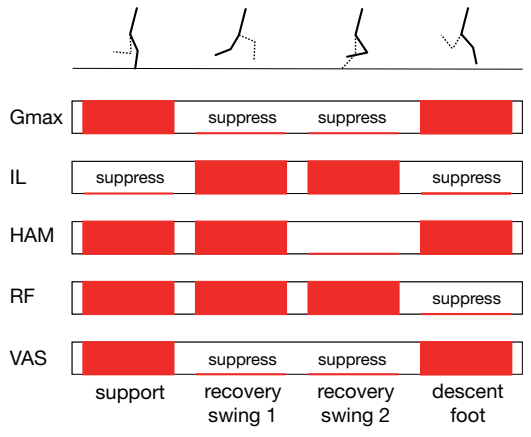

Fig. 9. The binalized activation pattern generated from human EMG data.

resulting end-effecter motion under the joint angles $\boldsymbol{\theta}$. The matrix $\boldsymbol{G}(\boldsymbol{\theta})$ is the Jacobian matrix represents the differential relationship between joint motion and the displacement of the muscle actuator. The each element of the matrix $\boldsymbol{G}(\boldsymbol{\theta})$ represents moment arms of the joint.

The required muscle tension forces $\boldsymbol{f}$ are determined from the desired force $\boldsymbol{Q}_{d}$ with numerical optimization eq.(5). We employ fatigue of the muscles weighted with the physiological cross section of the muscles as the evaluation function.

$$
\begin{array}{rl}
\min _{\boldsymbol{f}} & E=\sum_{i=1}^{n}\left(f_{i} / P_{i}\right)^{2} \\
\text { s.t. } & \boldsymbol{G}^{T}(\boldsymbol{\theta}) \boldsymbol{f}-\boldsymbol{J}^{T}(\boldsymbol{\theta}) \boldsymbol{Q}_{d}=\boldsymbol{O} \\
& f_{l b i} \leq f_{i} \leq f_{u b i} \quad i=1,2, \ldots, n
\end{array}
$$

The muscle tension $f$ must be set within a range of lower boundary $\boldsymbol{f}_{l b}$ to upper boundary $\boldsymbol{f}_{u b}$.

\section{Muscle Activation in the Aerial Phase}

The muscle activation patterns in the aerial phase are extracted from the human electromyographic (EMG) data and converted to the muscle activation for the robot. We can apply the human EMG data to the robot because the anatomical structure of the Athlete Robot is compatible with human.

In order to convert the signals, the EMG data is binalized as shown in Fig.9 with the threshold of 50\% of the peak value. Then, we employ the motor learning with random exploration and hill climbing method in physical simulator to determine the activation patterns. We use OpenHRP3 simulator which is free simulator with the efficient and precise computation method for the calculation of the dynamics. The binalized EMG data is used as the constraint of the muscle activation in the learning. The evaluation function is the forward distance of the robot. We transport the physical parameters of the robot from 3DCAD model to the OpenHRP3 simulator. The elastic blade of the foot is modeled as the translational joint. The characteristics of the pneumatic muscles are described with the theoretical equation [21]. Fig.10 shows acquired muscle activation pattern from binalized EMG data.

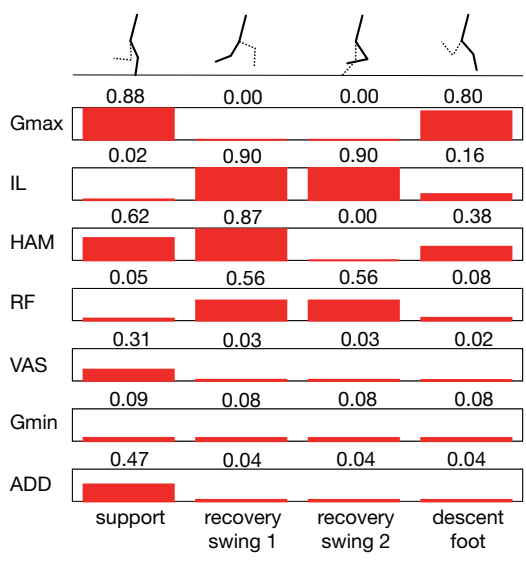

Fig. 10. The learned muscle activation pattern using dynamic simulation.
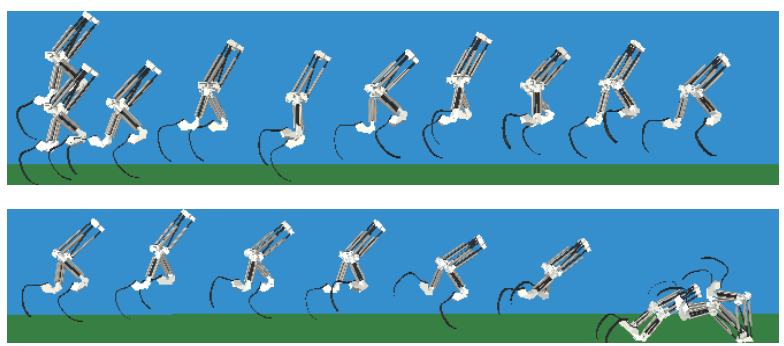

Fig. 11. The snapshot of the running simulation.
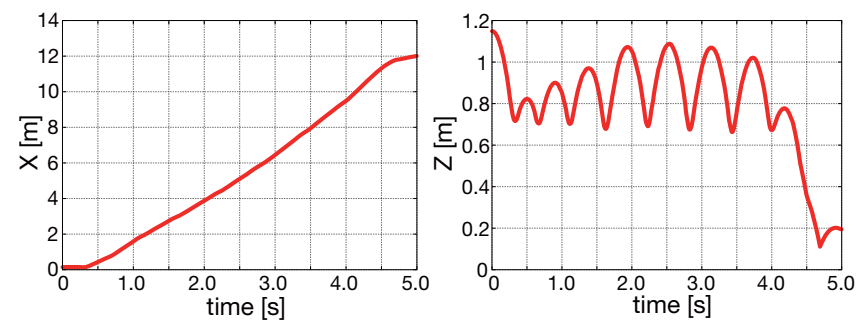

Fig. 12. CoG movement during eight step running.

\section{ROBOT EXPERIMENTS}

\section{A. Simulation Results}

We perform the bipedal running from the drop off and bouncing with given initial posture. The muscle activation pattern is generated from the reshaped human EMG as shown in Fig.10.

In the simulation experiment, we accomplish the eight steps running. Fig.12 shows the forward travel (x axis) and height ( $\mathrm{z}$ axis) of the center of gravity. The maximum velocity of the robot is about $3 \mathrm{~m} / \mathrm{s}(10 \mathrm{~km} / \mathrm{h})$. The robot fall down at a time of 4.5 second and reach a distance of about 12 meters. The Snapshots of the running are shown in Fig.11.

The average of total energy output of the muscles is calculated as $140.5 \mathrm{~J}$ per cycle. The average of energy output of the elastic blade foot from compressed state to the take off is about $18.0 \mathrm{~J}$. The results show that the elasticity of the blade foot reaches $12.8 \%$ of the total energy output of the muscles per cycle. 

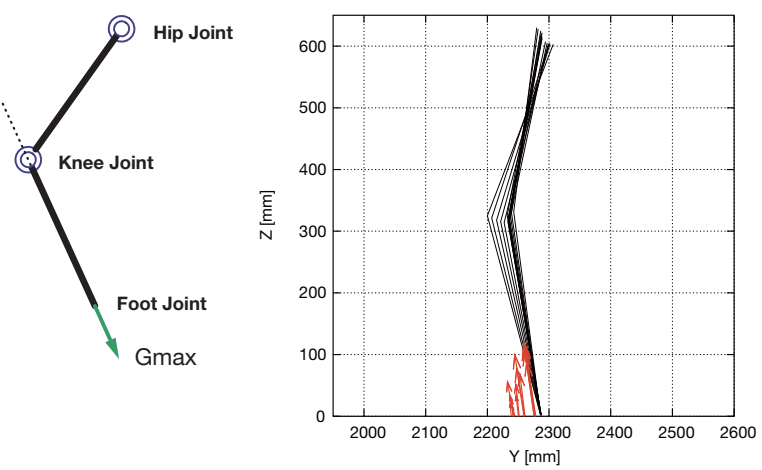

(a) theoretical force output

(b) measured reaction force

Fig. 13. Measured ground reaction force for the Gmax muscle.

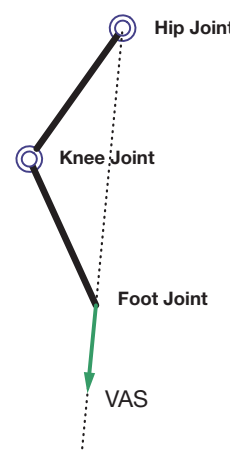

(a) theoretical force output

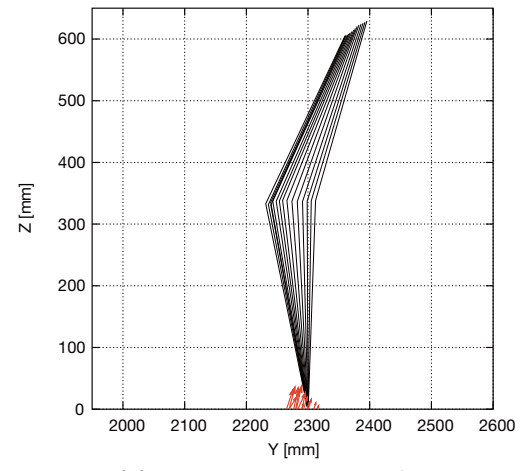

(b) measured reaction force
Fig. 14. Measured ground reaction force for the VAS muscle.

\section{B. Single Muscle Activation}

As a pilot study, we measured the ground reaction force corresponding to the activation of the single muscle using force plate. The robot is hanged from ceiling beam, and the leg is touch up to the force plate. We captured the motions using the Vicon motion capture system and Kistler force plates. The initial ground reaction force is reset to zero. Then we apply the pressure to a particular muscle. As shown in Fig.13 and Fig.14, measured ground reaction force consistent with the theoretical result. A vastus muscle (VAS) causes the forces to the hip joint and a Gluteus maximus (Gmax) muscle causes forces to the knee joint.

The results show that we can directly control the direction of the ground reaction force by the muscle activation.

\section{Running Experiment}

The initial position and posture of the robot is shown in Fig. 15 which corresponds with the simulation setup. The robot is hanged on a ceiling hook to sustain the posture. The robot is decoupled from the hook to start the running. The launch time is detected by the switch equipped in the hook. We use rubber suspender to prevent the collapse onto the floor.

The proposed musculoskeletal motor control method is experimentally tested on the Athlete Robot (Fig.17). We captured the motions using the Vicon motion capture system and Kistler force plates. The results show that the robot can run three steps

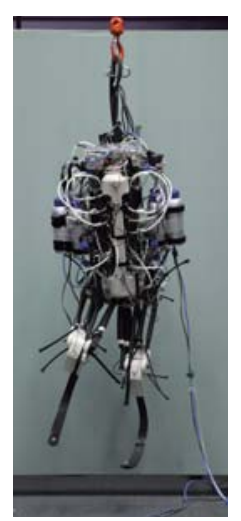

(a) front view

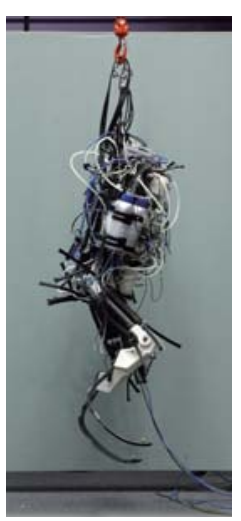

(b) side view
Fig. 15. Initial position and posture of the bipedal running.

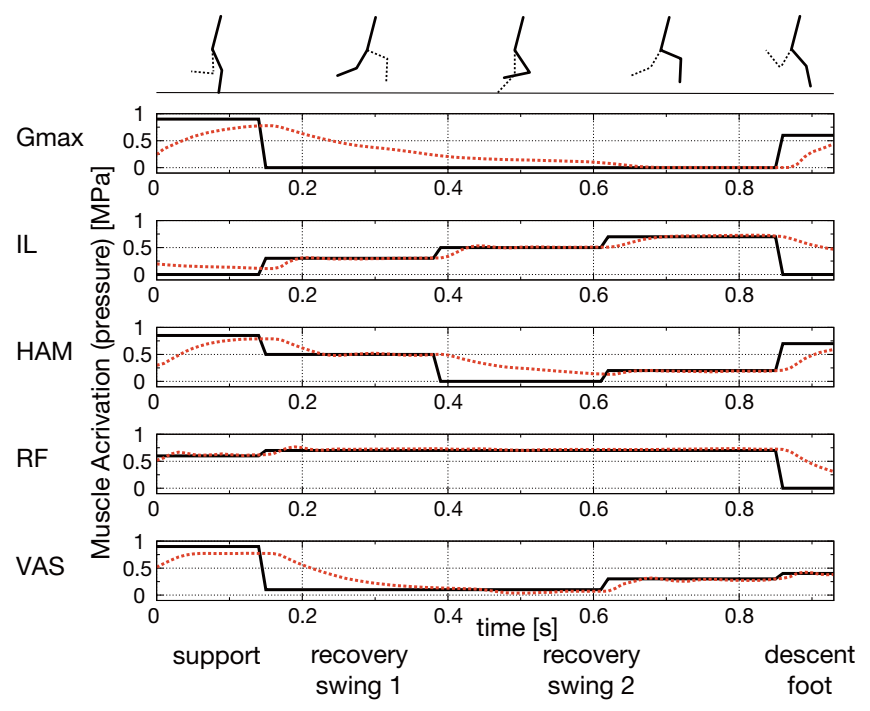

Fig. 16. The muscle activation patterns and measured innner pressure of the muscles during running.

$\mathrm{u}$

with artificial musculoskeletal system and elastic blade foot. The motor command is based on the activation patter used in simulation experiment and adjusted manually for the robot Fig.16. The robot uses two strides to move through 1.0 meters. The mean velocity of the robot is about $1.2 \mathrm{~m} / \mathrm{s}$. The energy losses in collision between blade foot and ground cause the decrease in performance from the simulation results.

\section{CONCLUSION}

We proposed the approach of musculoskeletal motor control for legged locomotion using muscle activity and kinetic data of the human movements. We demonstrate the bipedal running with musculoskeletal Athlete Robot with the elastic blade foot and pressure controlled pneumatic muscles. We use muscle activation patterns in support phase derived from physical measurement of human running and numerical optimization of the tension force of the muscles. We also apply the unique approach to determined the muscle activation in aerial phase based on the method that converting the human EMG signals 

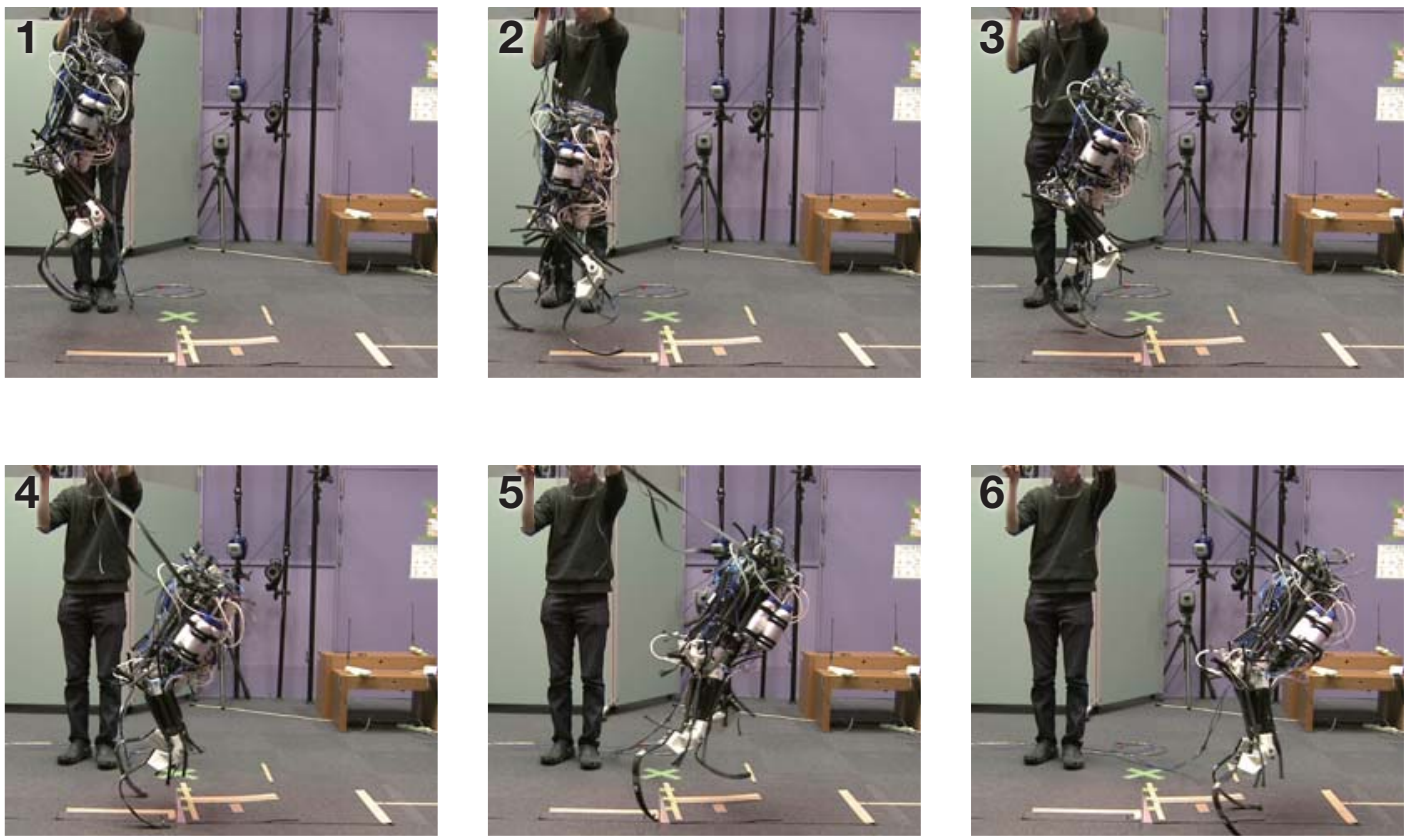

Fig. 17. Snapshots of the three steps of bipedeal running.

to the motor command of the robot. The future tasks are sensor feedback control to adjust motor command, and advanced analysis of measured movements of the robot.

\section{REFERENCES}

[1] Marc H. Raibert. Legged Robots That Balance. The MIT Press, 1986.

[2] Ryosuke Tajima, Daisaku Honda, and Keisuke Suga. Fast running experiments involving a humanoid robot. In Proc. IEEE Int. Conf. on Robotics and Automation (ICRA 2009), pages 1571-1576, may 2009.

[3] Toru Takenaka, Takashi Matsumoto, TakahideYoshiike, and Shinya Shirokura. Real time motion generation and control for biped robot -2nd report: Running gait pattern generation-. In Proc. IEEE/RSJ Int. Conf. on Intelligent Robots and Systems (IROS 2009), pages 1092 1099, October 10-15, 2009.

[4] R. McNeill Alexander and H. C. Bennet-Clark. Storage of elastic strain energy in muscle and other tissues. Nature, 265(5590):114-117, Jan 1977.

[5] Sang-Ho Hyon. Compliant terrain adaptation for biped humanoids without measuring ground surface and contact forces. IEEE Transactions on Robotics, 25(1):171-178, Feb. 2009.

[6] Marc Raibert, Kevin Blankespoor, Gabriel Nelson, Rob Playter, and the BigDog Team. Bigdog the rough-terrain quadruped robot. In Proceedings of the 17th World Congress, The International Federation of Automatic Control (IFAC), 2008.

[7] Ikuo Mizuuchi, Yuto Nakanishi, Yoshinao Sodeyama, Yuta Namiki, Tamaki Nishino, Naoya Muramatsu, Junichi Urata, Kazuo Hongo, Tomoaki Yoshikai, and Masayuki Inaba. An advanced musculoskeletal humanoid kojiro. In Proc. 7th IEEE-RAS Int. Conf. on Humanoid Robots (Humanoids 2007), pages 294-299, November 2007.

[8] Björn Verrelst, Ronald Van Ham, Bram Vanderborght, Frank Daerden, Dirk Lefeber, and Jimmy Vermeulen. The pneumatic biped "Lucy" actuated with pleated pneumatic artificial muscles. Autonomous Robots, 18(2):201-213, 2005.

[9] Koh Hosoda, Takashi Takuma, Atsushi Nakamoto, and Shinji Hayashi. Biped robot design powered by antagonistic pneumatic actuators for multi-modal locomotion. Robotics and Autonomous Systems, 56(1):4653, 2008.

[10] Caroline A. M. Doorenbosch and Gerrit Jan van Ingen Schenau. The role of mono- and bi-articular muscles during contact control leg tasks in man. Human Movement Science, 14(3):279-300, 1995.
[11] G. J. van Ingen Schenau, M. F. Bobbert, and R. H. Rozendal. The unique action of bi-articular muscles in complex movements. Journal of Anatomy, 155:1-5, Dec 1987

[12] Koji Ito, Toshio Tsuji, and Mitsuo Nagamachi. Motor impedance and inverse kinematics in musculoskeletal systems. In IEEE Engineering in Medicine \& Biology Society 10th Annual Int. Conf., pages 635-636, 1988.

[13] Toru Oshima, Tomohiko Fujikawa, Osamu Kameyama, and Minayori Kumamoto. Robotic analyses of output force distribution developed by human limbs. In IEEE Int. Workshop on Robot and Human Interactive Communication, 2000.

[14] M. D. Klein Horsman, H. F J M Koopman, F. C T van der Helm, L. Poliacu Pros, and H. E J Veeger. Morphological muscle and joint parameters for musculoskeletal modelling of the lower extremity. Clinical Biomechanics, 22(2):239-247, Feb 2007.

[15] Melissa G. Hoy, Felix E. Zajac, and Michael E. Gordon. A musculoskeletal model of the human lower extremity: the effect of muscle, tendon, and moment arm on the moment-angle relationship of musculotendon actuators at the hip, knee, and ankle. Journal of Biomechanics, 23(2):157-169, 1990.

[16] Tetsuo Fukunaga, Keitaro Kubo, Yasuo Kawakami, Senshi Fukashiro, Hiroaki Kanehisa, and Constantinos N. Maganaris. In vivo behaviour of human muscle tendon during walking. Proc. of the Royal Society B: Biological Sciences, 268(1464):229-233, Feb 2001.

[17] G. A. Lichtwark and A. M. Wilson. In vivo mechanical properties of the human achilles tendon during one-legged hopping. Journal of Experimantal Biology, 208(Pt 24):4715-4725, Dec 2005.

[18] G.A. Pratt and M.M. Williamson. Series elastic actuators. In Proc. IEEE/RSJ International Conference on Intelligent Robots and Systems 95, volume 1, pages 399-406, aug. 1995.

[19] Ryuma Niiyama, Kei Kakitani, and Yasuo Kuniyoshi. Learning to jump with a musculoskeletal robot using a sparse coding of activation. In Proc. ICRA 2009 Workshop on Approaches to Sensorimotor Learning on Humanoid Robots, pages 30-31, Kobe, Japan, May 2009.

[20] G. Cappellini, Y. P. Ivanenko, R. E. Poppele, and F. Lacquaniti. Motor patterns in human walking and running. Journal of Neurophysiology, 95(6):3426-3437, Jun 2006.

[21] Hal. F. Schulte, D. F. Adamski, and J. R. Pearson. Characteristics of the braided fluid actuator. Technical Report No.5, The University of Michigan Medical School Department of Physical Medicine and Rehabilitation Orthetics Research Project, 1961. 